\title{
Characterizations of Some Special Curves in Lorentz-Minkowski Space
}

\author{
M. Khalifa Saad ${ }^{1,2, *}$, R. A. Abdel-Baky ${ }^{3,4}$, F. Alharbi ${ }^{1}$, A. Aloufi ${ }^{1}$ \\ ${ }^{1}$ Department of Mathematics, Faculty of Science, Islamic University of Madinah, 170 Al-Madinah, KSA \\ ${ }^{2}$ Department of Mathematics, Faculty of Science, Sohag University, 82524 Sohag, Egypt \\ ${ }^{3}$ Department of Mathematics, Sciences Faculty for Girls, Jeddah University, 21352 Jeddah, KSA \\ ${ }^{4}$ Department of Mathematics, Faculty of Science, Assiut University, 71516 Assiut, Egypt
}

Received December 28, 2019; Revised March 10, 2020; Accepted March 19, 2020

Copyright $@ 2020$ by authors, all rights reserved. Authors agree that this article remains permanently open access under the terms of the Creative Commons Attribution License 4.0 International License

\begin{abstract}
In a theory of space curves, especially, a helix is the most elementary and interesting topic. A helix, moreover, pays attention to natural scientists as well as mathematicians because of its various applications, for example, DNA, carbon nanotube, screws, springs and so on. Also there are many applications of helix curve or helical structures in Science such as fractal geometry, in the fields of computer aided design and computer graphics. Helices can be used for the tool path description, the simulation of kinematic motion or the design of highways, etc. The problem of the determination of parametric representation of the position vector of an arbitrary space curve according to the intrinsic equations is still open in the Euclidean space $E^{3}$ and in the Minkowski space $E_{1}^{3}$. In this paper, we introduce some characterizations of a non-null slant helix which has a spacelike or timelike axis in $E_{1}^{3}$. We use vector differential equations established by means of Frenet equations in Minkowski space $E_{1}^{3}$. Also, we investigate some differential geometric properties of these curves according to these vector differential equations. Besides, we illustrate some examples to confirm our findings.
\end{abstract}

Keywords Frenet Formulae, Spacelike Curve, Timelike Curve, Position Vector, Slant Helix.

\section{Introduction}

The curves are a fundamental structure of differential geometry. In the differential geometry of a regular curve in the Euclidean 3-space $E^{3}$, it is well-known that one of the important problem is the characterization of a regular curve [1-5]. One of the interesting characteristics of such curves is that the ratio $\tau / \kappa$ is a constant, where $\tau$ and $\kappa$ stands for the torsion and curvature of the curve, respectively. A curve of constant slope or general helix is defined by the property that, the tangent lines make a constant angle with a fixed direction. A necessary and sufficient condition that a curve to be general helix in Minkowski space $E_{1}^{3}$ is that ratio of curvature to torsion be constant. Indeed, a helix is a special case of the general helix. If both curvature and torsion are non-zero constants, it is called a helix or a W-curve [2-8].

The study of this curve in $E^{3}$ dates from 1802 when Lancret stated that "a curve is a helix if and only if the ratio $\tau / \kappa$ is constant along the curve"[9]. Intuitively, the helix grows linearly in the direction of the axis. And also, it is known that a space curve $\alpha$ whose normal lines make a constant angle with a fixed direction is called a slant helix $[6,10]$. We remark that the principal normal lines of a cylindrical helix is perpendicular to fixed direction, so that a cylindrical helix is a slant helix. Helices arise in nanosprings, carbon nanotubes, $\alpha$-helices, DNA double and collagen triple helix, the double helix shape is commonly associated with DNA, since the double helix is structure of DNA [1-7]. They constructed a molecular model of DNA in which there were two complementary, antiparallel (side-by-side in opposite directions) strands of the bases guanine, adenine, thymine and cytosine, covalently linked through phosphodiesterase bonds (for details, see [2-12]).

In this work, we use vector differential equations established by means of Serret-Frenet formulae to give some characterizations of the above mentioned classes of curves that lie fully in Minkowski 3-space $E_{1}^{3}$. We hope our results will be helpful to mathematicians who are specialized on mathematical modeling.

\section{Preliminaries}

To meet the requirements in the next sections, here, the basic elements of the theory of curves in the Minkowski 3-space $E_{1}^{3}$ are briefly presented. There exists a vast literature on the sub- 
ject including several monographs(see for example [13-24]). Let $E_{1}^{3}$ be the three-dimensional Minkowski space, that is, the three-dimensional real vector space $R^{3}$ with the metric

$$
\langle d \mathbf{x}, d \mathbf{x}\rangle=d x_{1}^{2}+d x_{2}^{2}-d x_{3}^{2},
$$

where $\left(x_{1}, x_{2}, x_{3}\right)$ denotes the canonical coordinates in $R^{3}$. An arbitrary vector $\mathbf{x}$ of $E_{1}^{3}$ is said to be spacelike if $\langle\mathbf{x}, \mathbf{x}\rangle>0$ or $\mathbf{x}=\mathbf{0}$, timelike if $\langle\mathbf{x}, \mathbf{x}\rangle<0$ and lightlike or null if $\langle\mathbf{x}, \mathbf{x}\rangle=0$ and $\mathbf{x} \neq \mathbf{0}$. A timelike or light-like vector in $E_{1}^{3}$ is said to be causal. For $\mathbf{x} \in E_{1}^{3}$ the norm is defined by $\|\mathbf{x}\|=\sqrt{|\langle\mathbf{x}, \mathbf{x}\rangle|}$, then the vector $\mathbf{x}$ is called a spacelike unit vector if $\langle\mathbf{x}, \mathbf{x}\rangle=1$ and a timelike unit vector if $\langle\mathbf{x}, \mathbf{x}\rangle=-1$. Similarly, a regular curve in $E_{1}^{3}$ can locally be spacelike, timelike or null (lightlike), if all of its velocity vectors are spacelike, timelike or null (lightlike), respectively. For any two vectors $\mathbf{x}=\left(x_{1}, x_{2}, x_{3}\right)$ and $\mathbf{y}=\left(y_{1}, y_{2}, y_{3}\right)$ of $E_{1}^{3}$, the inner product is the real number $\langle\mathbf{x}, \mathbf{y}\rangle=x_{1} y_{1}+x_{2} y_{2}-x_{3} y_{3}$ and the vector product is defined by

$$
\mathbf{x} \times \mathbf{y}=\left(\left(x_{2} y_{3}-x_{3} y_{2}\right),\left(x_{3} y_{1}-x_{1} y_{3}\right),-\left(x_{1} y_{2}-x_{2} y_{1}\right)\right) .
$$

Many features of inner product spaces have analogues in the Minkowski space. For example, in the Euclidean space $\mathrm{E}^{3}$ the Schwarz inequality permits the definition of the Euclidean angle between two vectors $\mathbf{x}$ and $\mathbf{y}$ as the unique number $0 \leq \theta \leq \pi$ such that $\langle\mathbf{x}, \mathbf{y}\rangle=\|\mathbf{x}\|\|\mathbf{y}\| \cos \theta$. In the Minkowski space $E_{1}^{3}$ we have the following: Let us consider two non-null vectors $\mathbf{x}$ and $\mathbf{y} \in E_{1}^{3}$ such that they span a timelike plane $E_{1}^{2}$; in this plane we can consider an orthonormal basis $\{\mathbf{i}, \mathbf{j}\}$, with $\langle\mathbf{i}, \mathbf{i}\rangle=1$, and $\langle\mathbf{j}, \mathbf{j}\rangle=-1$. Vectors $\mathbf{x}$ and $\mathbf{y}$ can be written in this basis as $\mathbf{x}=x_{1} \mathbf{i}+x_{2} \mathbf{j}$ and $\mathbf{y}=y_{1} \mathbf{i}+y_{2} \mathbf{j}$.

Definition 1. Let us consider two non-null vectors $\mathbf{x}$ and $\mathbf{y}$ in $E_{1}^{3}$ [11-14].

(i) Let us assume that $\mathbf{x}$ and $\mathbf{y}$ are spacelike vectors, then

- If they span a spacelike plane; there is s a unique real number $0 \leq \theta \leq \pi$ such that

$\langle\mathbf{x}, \mathbf{y}\rangle=\|\mathbf{x}\|\|\mathbf{y}\| \cos \theta$. This number is called the spacelike angle between the vectors $\mathrm{x}$ and $\mathrm{y}$.

- If they span a timelike plane; there is s a unique real number $\theta \geq 0$ such that

$\langle\mathbf{x}, \mathbf{y}\rangle=\varepsilon\|\mathbf{x}\|\|\mathbf{y}\| \cosh \theta$, where $\varepsilon=+1$ or $\varepsilon=-1$ according to $\operatorname{sign}\left(x_{2}\right)=\operatorname{sign}\left(y_{2}\right)$ or $\operatorname{sign}\left(x_{2}\right) \neq \operatorname{sign}\left(y_{2}\right)$, respectively. This number is called the central angle between the vectors $\mathbf{x}$ and $\mathbf{y}$.

(ii) Let us assume that $\mathrm{x}$ and $\mathrm{y}$ are timelike vectors, then there is a unique real number $\theta \geq 0$ such that $\langle\mathbf{x}, \mathbf{y}\rangle=$ $\varepsilon\|\mathbf{x}\|\|\mathbf{y}\| \cosh \theta$, where $\varepsilon=+1$ or $\varepsilon=-1$ according to $\mathbf{x}$ and $\mathrm{y}$ have different time-orientation or the same time-orientation, respectively. This number is called the Lorentzian timelike angle between the vectors $\mathbf{x}$ and $\mathbf{y}$.

(iii) Let us assume that $\mathrm{x}$ is spacelike and $\mathrm{y}$ is timelike, then there is a unique real number $\theta \geq 0$ such that $\langle\mathbf{x}, \mathbf{y}\rangle=$ $\varepsilon\|\mathbf{x}\|\|\mathbf{y}\| \sinh \theta$, where $\varepsilon=+1$ or $\varepsilon=-1$ according to $\operatorname{sign}\left(x_{2}\right)=\operatorname{sign}\left(y_{1}\right)$ or $\operatorname{sign}\left(x_{2}\right) \neq \operatorname{sign}\left(y_{1}\right)$. This number is called the Lorentzian timelike angle between the vectors $\mathbf{x}$ and $\mathbf{y}$.
Let $\alpha=\alpha(s)$ be a unit speed non-null curve in $E_{1}^{3}$ has a spacelike or timelike rectifying plane; by $\kappa(s)$ and $\tau(s)$ we denote the natural curvature and torsion of $\alpha=\alpha(s)$, respectively. Consider the Serret-Frenet frame $\{\mathbf{t}(s), \mathbf{n}(s), \mathbf{b}(s)\}$ associated with curve $\alpha=\alpha(s)$, then the Frenet formulae read [5-9]:

$$
\left(\begin{array}{l}
\mathbf{t}^{\prime} \\
\mathbf{n}^{\prime} \\
\mathbf{b}^{\prime}
\end{array}\right)=\left(\begin{array}{lll}
0 & \kappa & 0 \\
-\epsilon_{0} \epsilon_{1} \kappa & 0 & \tau \\
0 & -\epsilon_{1} \epsilon_{2} \tau & 0
\end{array}\right)\left(\begin{array}{l}
\mathbf{t} \\
\mathbf{n} \\
\mathbf{b}
\end{array}\right),{ }^{\prime}=\frac{d}{d s},
$$

where $\epsilon_{0}=\langle\mathbf{t}, \mathbf{t}\rangle= \pm 1, \epsilon_{1}=\langle\mathbf{n}, \mathbf{n}\rangle= \pm 1, \epsilon_{2}=\langle\mathbf{b}, \mathbf{b}\rangle= \pm 1$, $\epsilon_{0} \epsilon_{1} \epsilon_{2}=-1$.

Let $\mathbf{m}$ be a fixed point in $E_{1}^{3}$. Then

$$
S_{1}^{2}(1)=\left\{\mathbf{x} \in E_{1}^{3} \mid\|\mathbf{x}-\mathbf{m}\|^{2}=1\right\},
$$

is the Lorentzian unit sphere and

$$
H_{+}^{2}(1)=\left\{\mathbf{x} \in E_{1}^{3} \mid\|\mathbf{x}-\mathbf{m}\|^{2}=-1\right\},
$$

is the hyperbolic unit sphere.

\section{Characterizations of Slant Helix}

It is important to point out that, in contrast to what happens in Euclidean space, in Minkowski ambient space we can not define the angle between two vectors (except that both vectors are of timelike type). For this reason, we give the Minkowski version of the slant helix which has been defined in the Euclidean 3-space $E^{3}$ as a curve for which $\langle\mathbf{n}, \mathbf{u}\rangle$ is a nonzero constant, where $\mathbf{n}$ is the unit principal normal vector of the curve $\alpha=\alpha(s)$ [10].

\subsection{Spacelike Slant Helix with a Spacelike Axis}

Let us first we consider that $\alpha=\alpha(s)$ be a regular unit speed spacelike curve with a spacelike or timelike principal normal in $E_{1}^{3}$. Then for non zero constant a spacelike slant helix with a spacelike axis in $E_{1}^{3}$ is defined by

$$
\langle\mathbf{u}, \mathbf{n}\rangle=a=\text { const., }\langle\mathbf{u}, \mathbf{u}\rangle=1 .
$$

Theorem 1. Let $\alpha=\alpha(s)$ be a unit speed non-null curve in $E_{1}^{3}$ and $\kappa(s)>0$. Then the following statements hold:

(i) $\alpha(s)$ is a spacelike slant helix with a spacelike principal normal and a spacelike axis if and only if for some indefinite integrals of $\kappa$, and $\tau$, the function

$$
f(s)=\left(\int k d s\right)^{2}-\left(\int \tau d s\right)^{2},
$$

is a constant $b$. For any $0<\theta<\pi / 2$, if we let $a=\cos \theta$, then $b=\tan ^{2} \theta$.

(ii) $\alpha(s)$ is a spacelike slant helix with a timelike principal normal and a spacelike axis if and only if for some indefinite integrals of $\kappa$, and $\tau$, the function

$$
f(s)=\left(\int k d s\right)^{2}+\left(\int \tau d s\right)^{2},
$$

is a constant $b$. For any real number $\theta>0$, if we let $a=\sinh \theta$, then $b=\operatorname{coth}^{2} \theta$. 
Proof. By the assumption we have $(\langle\mathbf{u}, \mathbf{t}\rangle)^{\prime}=a k,(\langle\mathbf{u}, \mathbf{b}\rangle)^{\prime}=$ $a \tau$. Then we can write out the components of $\mathbf{u}$ in the following form:

$$
\mathbf{u}=a\left(\int k d s\right) \mathbf{t}(s)+a \mathbf{n}(s)+a\left(\int \tau d s\right) \mathbf{b}(s) .
$$

Case (i): Since $\langle\mathbf{u}, \mathbf{u}\rangle=1$, for some indefinite integrals of $\kappa$, and $\tau$, we obtain

$$
\left(1-a^{2}\right)=a^{2}\left[\left(\int k d s\right)^{2}-\left(\int \tau d s\right)^{2}\right] .
$$

This means that if $a=\cos \theta$, then $b=\tan ^{2} \theta$, as claimed. Conversely, let us assume that

$$
\left(\int k d s\right)^{2}-\left(\int \tau d s\right)^{2}=b .
$$

Then we have $b=\tan ^{2} \theta$. Let

$$
\mathbf{m}(s)=\cos \theta\left(\int k d s\right) \mathbf{t}+\cos \theta \mathbf{n}-\cos \theta\left(\int \tau d s\right) \mathbf{b} .
$$

By Frenet's equations and the assumption, we have

$$
\frac{d \mathbf{m}(s)}{d s}=\mathbf{0}
$$

Hence $\mathbf{m}(s)$ is a constant spacelike unit vector satisfying $\langle\mathbf{n}, \mathbf{u}\rangle=\cos \theta$.

Case (ii): By a similar procedure as in Case (i), we have

$$
\left(1+a^{2}\right)=a^{2}\left[\left(\int k d s\right)^{2}+\left(\int \tau d s\right)^{2}\right] .
$$

This means that if $a=\sinh \theta$, then $b=\operatorname{coth}^{2} \theta$, as well.

Conversely, let us assume that

$$
\left(\int k d s\right)^{2}+\left(\int \tau d s\right)^{2}=b .
$$

Then we have $b=\operatorname{coth}^{2} \theta$. Let

$$
\mathbf{m}(s)=-\sinh \theta\left(\int k d s\right) \mathbf{t}+\sinh \theta \mathbf{n}-\sinh \theta\left(\int \tau d s\right) \mathbf{b} .
$$

As stated in the above case, we have

$$
\frac{d \mathbf{m}(s)}{d s}=\mathbf{0}
$$

Hence $\mathbf{m}(s)$ is a constant spacelike unit vector satisfying $\langle\mathbf{n}, \mathbf{u}\rangle=\sinh \theta$.

\subsection{Spacelike Slant Helix with a Timelike Axis}

Now, we consider that $\alpha=\alpha(s)$ be a regular unit speed spacelike curve with a spacelike or timelike principal normal in $E_{1}^{3}$. Then for non zero constant a spacelike slant helix with a timelike axis in $E_{1}^{3}$ is defined by

$$
\langle\mathbf{u}, \mathbf{n}\rangle=a=\text { const. },\langle\mathbf{u}, \mathbf{u}\rangle=-1 \text {. }
$$

Theorem 2. Suppose that $\alpha(s)$ be a unit speed spacelike curve with $\kappa(s)>0$. Then the following statements hold:

i) $\alpha(s)$ is a spacelike slant helix with a spacelike principal normal and a timelike axis if and only if for some indefinite integrals of $\kappa$, and $\tau$, the function

$$
f(s)=-\left(\int k d s\right)^{2}+\left(\int \tau d s\right)^{2}
$$

is a constant $b$. For any $\theta>0$, if we let $a=\sinh \theta$, then $b=\operatorname{coth}^{2} \theta$.

ii) $\alpha(s)$ is a spacelike slant helix with a timelike principal normal and a timelike axis if and only if for some indefinite integrals of $\kappa$, and $\tau$, the function

$$
f(s)=\left(\int k d s\right)^{2}+\left(\int \tau d s\right)^{2},
$$

is a constant $b$. For any real number $\theta>0$, if we let $a=\cosh \theta$, then $b=\tanh ^{2} \theta$.

Proof. By the assumption we have $(\langle\mathbf{u}, \mathbf{t}\rangle)^{\prime}=a k,(\langle\mathbf{u}, \mathbf{b}\rangle)^{\prime}=$ $a \tau$. Then we can write out the components of $\mathbf{u}$ in the following form:

$$
\mathbf{u}=a\left(\int k d s\right) \mathbf{t}(s)+a \mathbf{n}(s)+a\left(\int \tau d s\right) \mathbf{b}(s) .
$$

Case (i): Since $\langle\mathbf{u}, \mathbf{u}\rangle=-1$, for some indefinite integrals of $\kappa$, and $\tau$, we obtain

$$
\left(1+a^{2}\right)=a^{2}\left[-\left(\int k d s\right)^{2}+\left(\int \tau d s\right)^{2}\right] .
$$

This means that if $a=\sinh \theta$, then $b=\operatorname{coth}^{2} \theta$, as claimed.

Conversely, let us assume that

$$
-\left(\int k d s\right)^{2}+\left(\int \tau d s\right)^{2}=b .
$$

Then we have $b=\operatorname{coth}^{2} \theta$. Let

$$
\mathbf{m}(s)=\sinh \theta\left(\int k d s\right) \mathbf{t}+\sinh \theta \mathbf{n}-\sinh \theta\left(\int \tau d s\right) \mathbf{b} .
$$

By the assumption we have

$$
\frac{d \mathbf{m}(s)}{d s}=\mathbf{0} .
$$

Hence $\mathbf{m}(s)$ is a constant timelike unit vector satisfying $\langle\mathbf{n}, \mathbf{u}\rangle=\sinh \theta$.

Case (ii): As stated in the above case, we have

$$
\left(-1+a^{2}\right)=a^{2}\left[\left(\int k d s\right)^{2}+\left(\int \tau d s\right)^{2}\right] .
$$

This means that if $a=\cosh \theta$, then $b=\tanh ^{2} \theta$.

Conversely, let us assume that

$$
\left(\int k d s\right)^{2}+\left(\int \tau d s\right)^{2}=b .
$$


Then we have $b=\tanh ^{2} \theta$. Let

$$
\mathbf{m}(s)=\cosh \theta\left(\int k d s\right) \mathbf{t}-\cosh \theta \mathbf{n}+\cosh \theta\left(\int \tau d s\right) \mathbf{b} .
$$

By the assumption we have

$$
\frac{d \mathbf{m}(s)}{d s}=\mathbf{0}
$$

Hence $\mathbf{m}(s)$ is a constant timelike unit vector satisfying $\langle\mathbf{n}, \mathbf{u}\rangle=\cosh \theta$.

Case (ii): Since $\langle\mathbf{u}, \mathbf{u}\rangle=1$, for some indefinite integrals of $\kappa$, and $\tau$, we obtain

$$
1-a^{2}=a^{2}\left[-\left(\int k d s\right)^{2}+\left(\int \tau d s\right)^{2}\right] .
$$

This means that if $a=\cos \theta$, then $b=\tan ^{2} \theta$.

Conversely, let us assume that

$$
-\left(\int k d s\right)^{2}+\left(\int \tau d s\right)^{2}=b .
$$

Then we have $b=\tan ^{2} \theta$. Let

$$
\mathbf{m}(s)=-\cos \theta\left(\int k d s\right) \mathbf{t}+\cos \theta \mathbf{n}-\cos \theta\left(\int \tau d s\right) \mathbf{b} .
$$

By Serret-Frenet formulae and the assumption, we have

$$
\frac{d \mathbf{m}(s)}{d s}=\mathbf{0} .
$$

Hence $\mathbf{m}(s)$ is a constant spacelike unit vector satisfying $\langle\mathbf{n}, \mathbf{u}\rangle=\cos \theta$.

\subsection{Timelike Slant Helix with a Spacelike or Timelike Axis}

Here, We consider that $\alpha=\alpha(s)$ be a regular unit speed timelike curve with a spacelike or timelike axis in $E_{1}^{3}$. Then for non zero constant a timelike slant helix with a spacelike or timelike axis in $E_{1}^{3}$ is defined by

$$
\langle\mathbf{u}, \mathbf{n}\rangle=-a=\text { const. },
$$

where $\mathbf{u}$ is a spacelike or timelike axis of $\alpha(s)$.

Theorem 3. Let $\alpha=\alpha(s)$ be a unit speed timelike curve in $E_{1}^{3}$ with $\kappa(s)>0$. Then the following statements hold:

(i) $\alpha(s)$ is a timelike slant helix with a spacelike axis if and only if for some indefinite integrals of $\kappa$, and $\tau$, the function

$$
f(s)=-\left(\int k d s\right)^{2}+\left(\int \tau d s\right)^{2},
$$

is a constant $b$. For any $0<\theta<\pi / 2$, if we let $a=\cos \theta$, then $b=\tan ^{2} \theta$.

(ii) $\alpha(s)$ is a timelike slant helix with a timelike axis if and only if for some indefinite integrals of $\kappa$, and $\tau$, the function

$$
f(s)=\left(\int k d s\right)^{2}-\left(\int \tau d s\right)^{2},
$$

is a constant $b$. For any real number $\theta>0$, if we let $a=\sinh \theta$, then $b=\operatorname{coth}^{2} \theta$.
Proof. By the assumption we have $(\langle\mathbf{u}, \mathbf{t}\rangle)^{\prime}=$ $-a k,(\langle\mathbf{u}, \mathbf{b}\rangle)^{\prime}=a \tau$. Then we can write out the components of $\mathbf{u}$ in the following form:

$$
\mathbf{u}=-a\left(\int k d s\right) \mathbf{t}(s)-a \mathbf{n}(s)+a\left(\int \tau d s\right) \mathbf{b}(s) .
$$

Case (i): Since $\langle\mathbf{u}, \mathbf{u}\rangle=1$, for some indefinite integrals of $\kappa$, and $\tau$, we obtain

$$
1-a^{2}=a^{2}\left[-\left(\int k d s\right)^{2}+\left(\int \tau d s\right)^{2}\right] .
$$

This means that if $a=\cos \theta$, then $b=\tan ^{2} \theta$, as claimed. Conversely, let us assume that

$$
-\left(\int k d s\right)^{2}+\left(\int \tau d s\right)^{2}=b .
$$

Then we have $b=\tan ^{2} \theta$. Let

$$
\mathbf{m}(s)=-\cos \theta\left(\int k d s\right) \mathbf{t}+\cos \theta \mathbf{n}-\cos \theta\left(\int \tau d s\right) \mathbf{b} .
$$

By Serret-Frenet formulae and the assumption, we have

$$
\frac{d \mathbf{m}(s)}{d s}=\mathbf{0} .
$$

Hence $\mathbf{m}(s)$ is a constant spacelike unit vector satisfying $\langle\mathbf{n}, \mathbf{u}\rangle=\cos \theta$.

Case (ii): By a similar procedure as in Case (i), we have

$$
\left(1+a^{2}\right)=a^{2}\left[\left(\int k d s\right)^{2}-\left(\int \tau d s\right)^{2}\right] .
$$

This means that if $a=\sinh \theta$, then $b=\operatorname{coth}^{2} \theta$.

Conversely, let us assume that

$$
\left(\int k d s\right)^{2}-\left(\int \tau d s\right)^{2}=b .
$$

Then we have $b=\operatorname{coth}^{2} \theta$. Let

$$
\mathbf{m}(s)=-\sinh \theta\left(\int k d s\right) \mathbf{t}+\sinh \theta \mathbf{n}-\sinh \theta\left(\int \tau d s\right) \mathbf{b} .
$$

As stated in the above case, we have

$$
\frac{d \mathbf{m}(s)}{d s}=\mathbf{0} .
$$

Hence $\mathbf{m}(s)$ is a constant timelike unit vector satisfying $\langle\mathbf{n}, \mathbf{u}\rangle=\sinh \theta$.

\subsection{Example}

Now, we give an example of a spacelike and timelike slant helix in Minkowski 3-space $E_{1}^{3}$ and illustrate its pictures by using Mathematical program.

Let us first consider the following explicit parametric representation of a spacelike slant helix of $E_{1}^{3}$ :

$$
x(u)=\left(x_{1}(u), x_{2}(u), x_{3}(u)\right),
$$


where

$$
\begin{aligned}
x_{1}(u) & =\frac{e}{f} \sin \nu u, \\
x_{2}(u) & =\frac{a}{b} \sin \lambda u+\frac{c}{d} \sin \mu u, \\
x_{3}(u) & =-\frac{a}{b} \cos \lambda u+\frac{c}{d} \cos \mu u,
\end{aligned}
$$

where $a, b, c, d, e, f, \lambda, \mu, \nu$ are constants. Since its position vector is a spacelike vector, it lies on the pseudo-sphere (see Fig. 1 and Fig. 2).

And we also consider the explicit parametric representation of a timelike slant helix:

$$
y(v)=\left(y_{1}(v), y_{2}(v), y_{3}(v)\right)
$$

where

$$
\begin{aligned}
y_{1}(v) & =\frac{a}{b} \sinh \lambda v+\frac{c}{d} \sinh \mu v \\
y_{2}(v) & =\frac{a}{b} \cosh \lambda v-\frac{c}{d} \cosh \mu v \\
y_{3}(v) & =\frac{e}{f} \sinh \nu v
\end{aligned}
$$

So, the curve lies on the hyperboloid of one sheet (see Fig. 3 and Fig. 4).

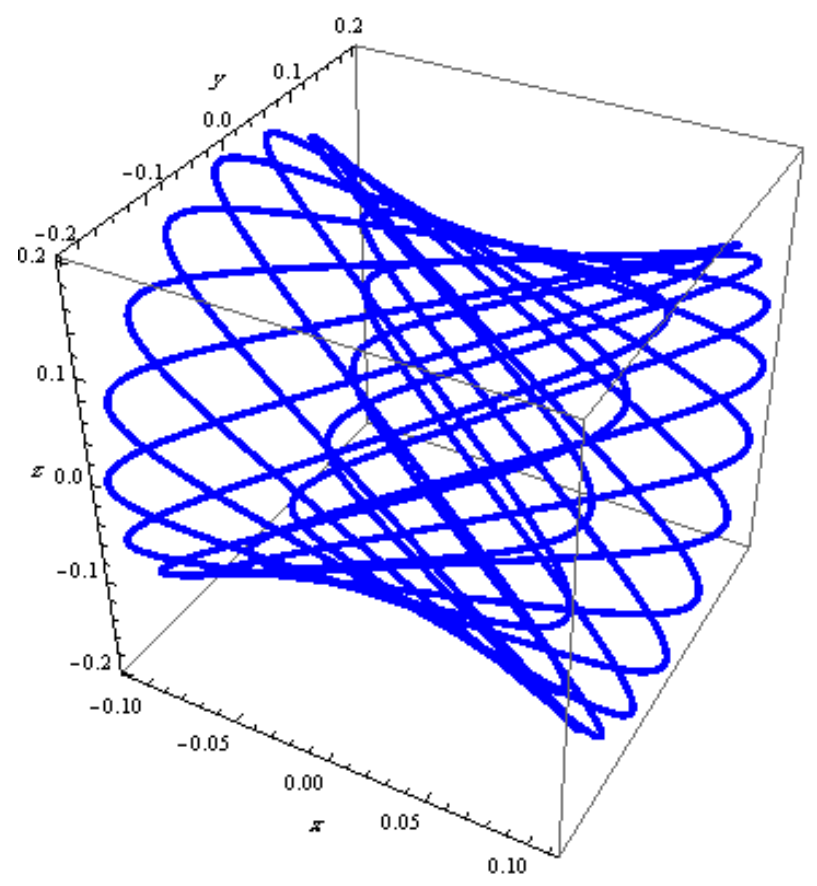

Figure 1. The spacelike slant helix $x(u)$.

\section{Conclusions}

In the three-dimensional Minkowski space, some characterizations of a spacelike and timelike slant helix have a non-null axis are introduced. By using vector differential equations established by means of Serret-Frenet equations in Minkowski space $E_{1}^{3}$, the differential geometric properties of these curves

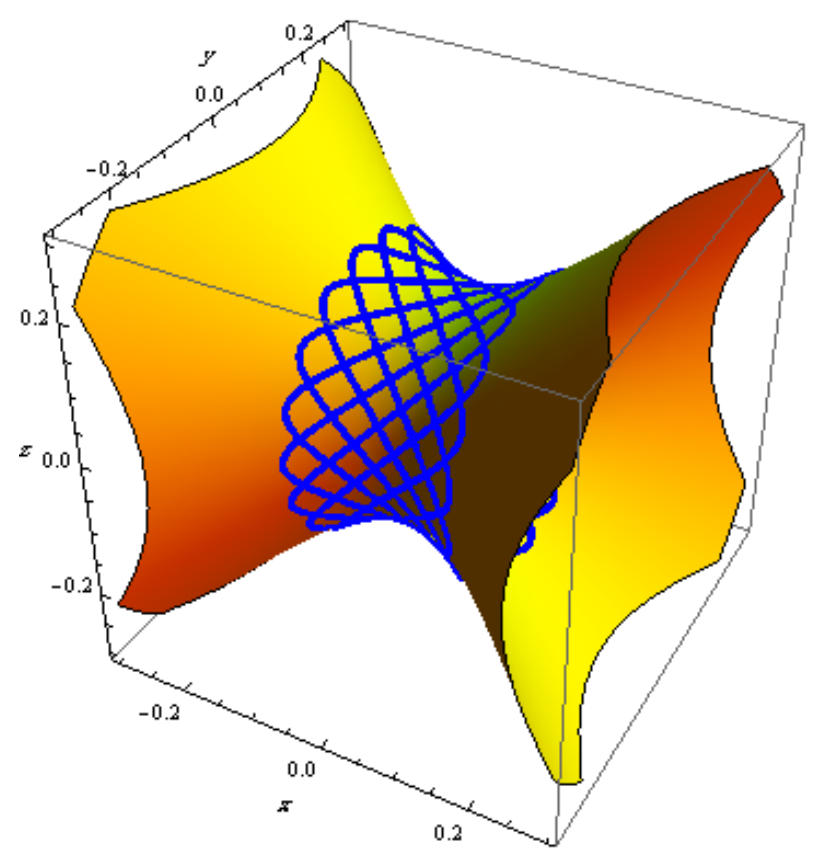

Figure 2. $x(u)$ lies on the pseudo-sphere.

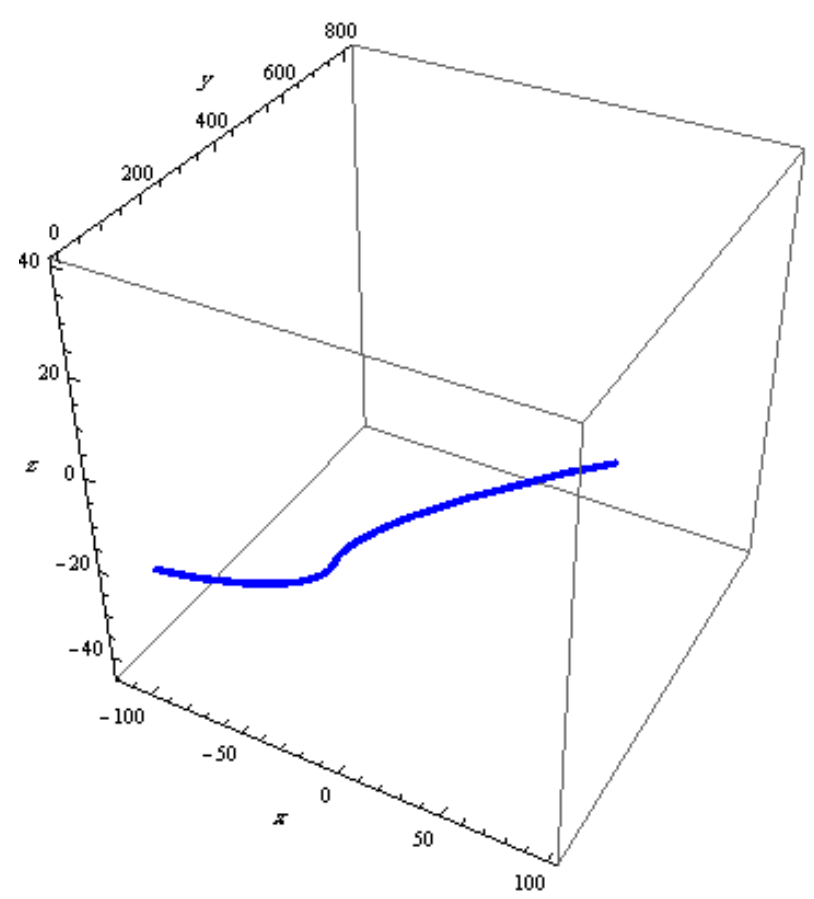

Figure 3. The timelike slant helix $y(v)$. 


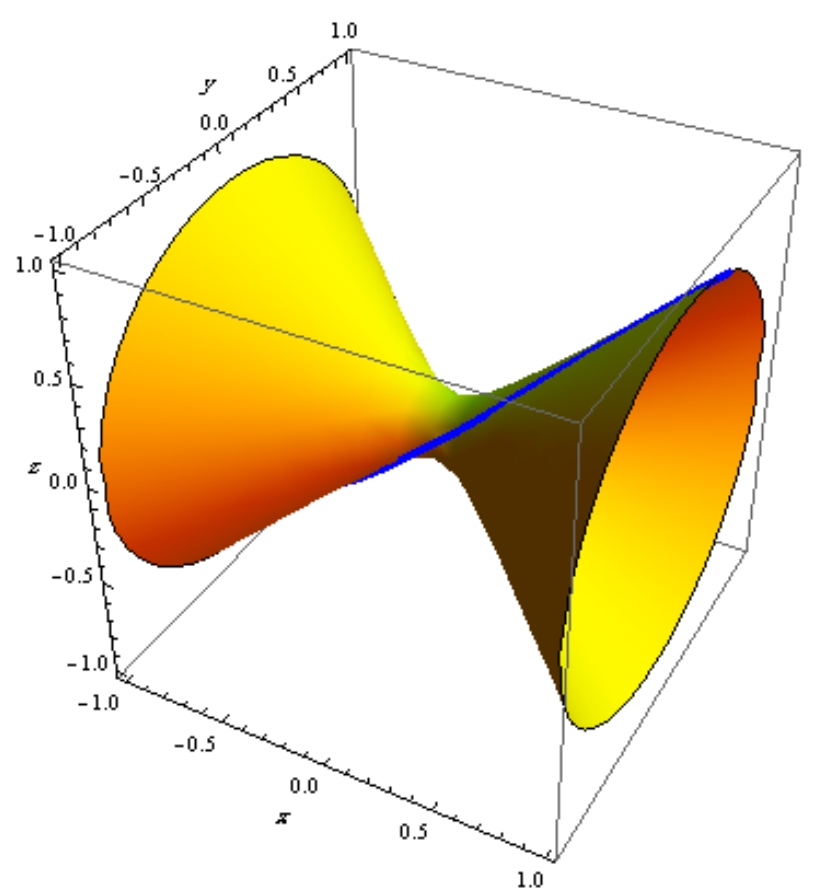

Figure 4. $y(v)$ lies on the hyperboloid of one sheet.

are investigated. Finally, by using Wolfram Mathematica 0.7, an example for a spacelike and timelike slant helix is given and illustrated.

\section{Acknowledgements}

This research was supported by Islamic University of Madinah. We would like to thank our colleagues from Deanship of Scientific Research who provided insight and expertise that greatly assisted the research.

\section{REFERENCES}

[1] S. K. Nurkan, Y. A. Gven, M. K. Karacan, Characterizations of adjoint curves in euclidean 3-space. Proc. Natl. Acad. Sci., India, Sect. A Phys. Sci., 2018.

[2] M. Yavari, M. Zarrati, The slant helix solutions of the equilibrium shape equations for the biopolymer chains. Chinese J. Phys. vol. 55, pp. 444-456, 2017.

[3] M. Bektas, M. Kulahci, Differential equations characterizing spacelike curves in the 3- dimensional lightlike cone, Palest. J. Math., vol. 6, no. 2, pp. 330?37, 2017.

[4] N. Abazari, M. Bohner, I. Sager, Y. Yayli, Stationary acceleration of Frenet curves, J. Inequal. Appl., no. 92, 13 pages, 2017.

[5] B. Bukcu, M. K. Karacan, Spherical curves with modified orthogonal frame. J. New Res. Sci., vol. 10, pp. 60-68, 2016.
[6] J. W. Lee, No null-helix Mannheim curves in the Minkowski space E31. Int. J. Math. Math. Sci. vol. 7, pp. 44?07. 2014.

[7] R. Lopez, Differential geometry of curves and surfaces in Lorentz-Minkowski space, Int. Electron. J. Geom., vol. 7, no. 1, pp. 44?07, 2014.

[8] T.A. Ahmad, R. Lopez, Slant helices in minkowski space E31. J. Korean Math. Soc. vol. 48, pp. 159-167, 2011.

[9] H. Liu, Q. Meng, Representation formulas of curves in a twoand three-dimensional lightlike cone, Results Math., vol. 59, no. 3-4, pp. 437?51, 2011.

[10] C. Camc., K. İlarslan, L. Kula, H.H. Hacsıalihoğlu, Harmonic curvatures and generalized helices in En, Chaos Solitons Fractals, vol. 4, pp. 2590-2596, 2009.

[11] M. Turgut, S. Yilmaz, Contributions to classical differential geometry of the curves in $E^{3}$, Sci. Magna., vol. 4, pp. 5-9, 2008.

[12] K. İlarslan, Ö. Boyacoğlu, Position vectors of a timelike and a null helix in Minkowski 3-space, Chaos Solitons Fractals, vol. 38, pp. 1383-1389, 2008.

[13] K. İlarslan, O. Boyacoğlu, Position vectors of a space-like W-curve in Minkowski space, Bull. Korean Math. Soc., vol. 44, pp. 429-438, 2007.

[14] N. Chouaieb, A. Goriely, J. H. Maddocks, Helices, Proc. Natl. Acad. Sci. USA, vol. 103, pp. 398-403, 2006.

[15] K. İlarslan, Spacelike normal curves in Minkowski space $E_{1}^{3}$, Turkish J. Math., vol. 29, pp. 53-63, 2005.

[16] Kula, L., Yayl? Y.: On slant helix and its spherical indicatrix. Appl. Math. Comput. 169 (2005), 600-607.

[17] S. Izumiya and N. Takeuchi, New special curves and developable surfaces, Turkish J. Math., vol. 28, pp. 153-163, 2004.

[18] K. İlarslan, E. Nesovic, M. Petrovic-Torgasev, Some characterizations of rectifying curves in the Minkowski 3-space, Novi Sad J Math., vol. 33, no. 2, pp. 23-32, 2003.

[19] A. Ferrandez, A. Gimenez, P. Lucas, Null helices in Lorentzian space forms, Internat. J. Modern Phys. A, vol. 16, pp. 48454863, 2001.

[20] J. Walfare, Curves and Surfaces in Minkowski Space, Ph.D. Thesis, K.U. Leuven, Faculty of Science, Leuven, 1995.

[21] D.S. Kim, Chung and K.H. Cho, Space curves satisfying $\tau / \kappa=a s+b$, Honam Math. J., vol. 15, no. 1, pp. 5-9, 1993. 
[22] D. J. Struik, Lectures on Classical Differential Geometry, Dover, New-York, 1988.

[23] B. O'Neil, Semi-Riemannian Geometry geometry, with appli- cations to relativity, Academic Press, New York, 1983.

[24] T. A. Cook, The Curves of Life, Constable, London, 1914, reprinted, Dover, London, 1979. 